Boletín de la Sociedad Zoológica del Uruguay, 2020

Vol. 29 (2): 126-131

ISSN 2393-6940

https://journal.szu.org.uy

\title{
CONSTANCIA FLORAL EN LOS ABEJORROS NATIVOS Bombus pauloensis Y Bombus Bellicosus
}

\author{
Sheena Salvarrey ${ }^{1}$, Natalia Arbulo ${ }^{2}$, Estela Santos ${ }^{1}$, Ciro Invernizzi ${ }^{1}$ \\ 1 Sección Etología, Facultad de Ciencias, Udelar. Iguá 4225 (CP 11400), Montevideo, Uruguay. \\ 2 Centro Universitario Regional del Este, Udelar. Ruta nacional №9 intersección con ruta №15 (CP 27000), \\ Rocha, Uruguay.
}

Autor por correspondencia: ssalvarrey@ fcien.edu.uy

\section{RESUMEN}

La constancia floral que muestran los ápidos durante sus viajes de forrajeo es un tema de amplio debate, tanto por los mecanismos cognitivos subyacentes, como por su implicancia en la optimización en la recolección de alimento y por las consecuencias para las especies botánicas visitadas. Los abejorros del género Bombus han sido buenos modelos para estudiar este comportamiento. En este estudio se analizó la constancia floral de los abejorros nativos Bombus pauloensis y Bombus bellicosus en una pradera compuesta de trébol rojo, trébol blanco y lotus. Para ello se registraron las especies botánicas que visitaban los abejorros durante un tramo de su vuelo de forrajeo. Se encontraron diferencias interespecíficas en la constancia floral. El $70 \%$ de $B$. pauloensis y el $43,5 \%$ de $B$. bellicosus que visitaron al menos 20 unidades florales individuales (UFI = inflorescencia para tréboles y flores para lotus) visitaron un solo recurso $(\mathrm{P}<0.001)$. Al analizar los vuelos de UFI a UFI B. pauloensis presentó una constancia de vuelos de $98.7 \%$ y $B$. bellicosus de $94.4 \%(P<0.05)$. El grado de constancia floral que presentan los abejorros sería una respuesta adaptativa, donde están involucradas las restricciones cognitivas, la disponibilidad de recursos, la competencia con otras especies y la eficiencia en la extracción de néctar y el polen.

Palabras claves: Bombus, comportamiento, polinización

\section{ABSTRACT}

Floral constance in the native bees Bombus pauloensis and Bombus bellicosus. Flower constancy shown by the apids during their foraging trips is a subject of wide debate, both because of the underlying cognitive mechanisms, as for its implication in optimizing the food collection and for the consequences for the botanical species visited. Bumblebees of the genus Bombus have been good models for studying this behavior. In this study, the flower constancy of the native bumblebees Bombus pauloensis and Bombus bellicosus was analyzed in a meadow composed of red clover, white clover and bird's foot trefoil. To do this, the botanical species that bumblebees visited during a section of their foraging flight were recorded. Interspecific differences were found in flower constancy. Seventy percent of $B$. pauloensis and $43.5 \%$ of $B$. bellicosus that visited at least 20 individual flower units (IFU $=$ inflorescences for clovers and flowers for bird's foot trefoils) visited a single resource $(P<0.001)$. When analyzing the flights from IFU to IFU, B. pauloensis presented a flight constancy of $98.7 \%$ and $B$. bellicosus of $94.4 \%(P<0.05)$. The degree of flower constancy shown by bumblebees would be an adaptive response, where cognitive restrictions, resource availability, competition with other species and efficiency in the extraction of nectar and pollen are involved.

Key words: Bombus, behavior, pollination

\section{INTRODUCCIÓN}

Los abejorros del género Bombus (Hymenoptera; Apoidea) son insectos eusociales, con ciclo anual que cumplen un rol fundamental en el mantenimiento de las comunidades vegetales y en la producción agrícola (Goulson, 2003; Ashman et al., 2004; Michener, 2007; Klein et al., 2007). Uruguay cuenta con la presencia de dos especies nativas, Bombus pauloensis (Syn = $B$. atratus) y $B$. bellicosus. La primera está ampliamente distribuida por todo el territorio nacional mientras que la segunda se restringe al sur del Río Negro (Santos et al., 2017). Los abejorros presentan características morfológicas y comportamentales que los convierten en excelentes polinizadores, motivo por el cual en la actualidad han adquirido protagonismo en la actividad agrícola. En este sentido, a nivel nacional, se ha comprobado que pueden contribuir sustancialmente en la producción de cultivos comerciales tales como, el tomate (Solanum lycopersicum) y trébol rojo (Trifolium pratense) (Salvarrey et al., 2017; 2020). No obstante, el valor de estos abejorros nativos no se restringe a cultivos agrícolas sino que tienen un rol muy relevante en la conservación de la diversidad vegetal en general, ya que polinizan un gran número de especies vegetales silvestres (Arbulo et al., 2011; Rossi et al., 2015; Santos et al., 2017; Salvarrey, 2019). 


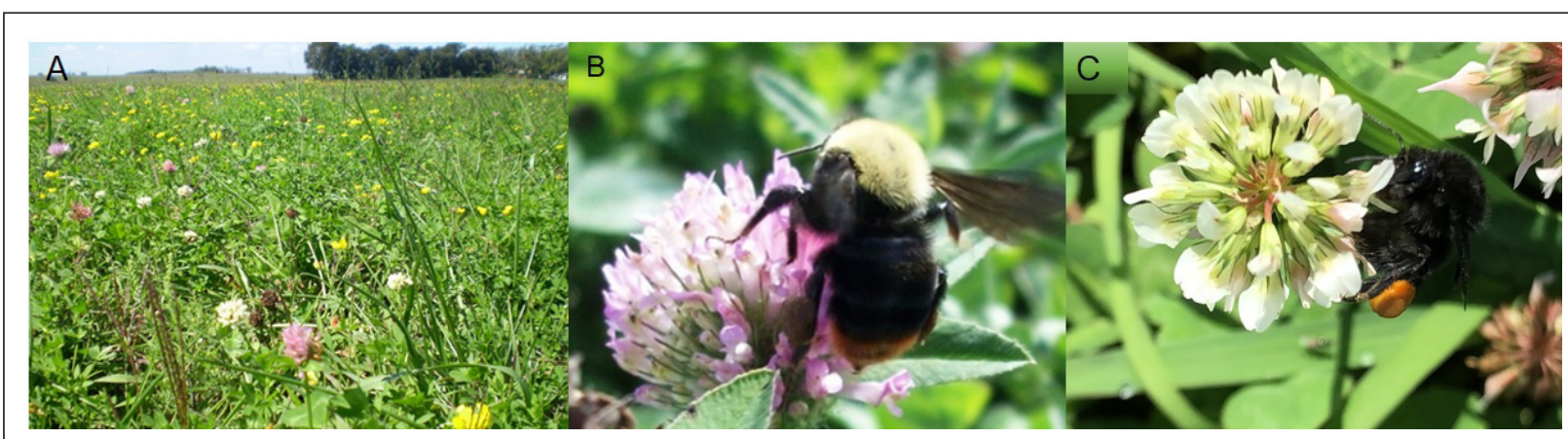

Fig. 1. Vista de la pradera donde se realizaron los seguimientos compuesta por trébol rojo, trébol blanco y lotus (A), Bombus bellicosus (B), Bombus pauloensis (C).

Los abejorros, al igual que otros polinizadores, presentan un comportamiento denominado constancia floral, el cual consiste en la tendencia a visitar una única especie vegetal durante su viaje de pecoreo (Free, 1970; Goulson, 2003). Desde el punto de vista de las plantas, la constancia floral sería beneficiosa ya que aseguraría la transferencia de polen entre plantas de la misma especie y por otro lado, disminuiría la competencia interespecífica entre plantas por el servicio de polinización, ya que los insectos muestran marcadas preferencias hacia determinadas especies (Chittka et al., 1999; Goulson, 2000; 2003). Para el polinizador, la utilización reiterada de una misma especie floral luego de evaluarla como la más redituable, sería una forma de optimizar la eficiencia de forrajeo, aún teniendo que eludir otros recursos que puedan ser igualmente recompensantes (Waser, 1986; Laverty, 1994; Wiegmann et al., 2003; Heinrich, 2004; Raine \& Chittka, 2007). Este comportamiento no responde a lo esperado por la teoría de Forrajeo Óptimo, ya que las pecoreadoras podrían disminuir el costo de volar a mayores distancias utilizando otros recursos cercanos de similar valor reduciendo la relación costo/ beneficio (Waser, 1986).

Se han propuesto diversas explicaciones a este comportamiento, una de ellas evoca una limitación en el aprendizaje que estaría relacionada con las diversas morfologías florales y las estrategias de extraer los recursos alimenticios (Waser, 1986; Laverty, 1994; Goulson, 2003; Heinrich, 2004; Raine \& Chittka, 2007). También se planteó que estos problemas de aprendizaje estarían íntimamente ligados con limitaciones en la memoria (Chittka et al., 1999; Grüter \& Ratnieks, 2011). Sin embargo, los abejorros pueden recordar cómo explotar exitosamente numerosos tipos florales, por lo que el problema estaría dado específicamente por la memoria a corto plazo (Chittka et al., 1999; Hill et al., 2001; Gegear \& Laverty, 2005). En esta memoria es donde se guardan las características de la flor con la que los abejorros las identifican. Sin embargo, si esta especie vegetal está en baja proporción, a los pocos segundos ese "recuerdo" se desvanece y la probabilidad de cambiar a otra especie floral aumenta (Raine \& Chittka, 2007). Básicamente, las limitaciones en el cerebro impedirían a corto plazo que los abejorros reconozcan un recurso y lo asocien correctamente con la recompensa que brindan y la forma de manipularlo (Raine \& Chittka, 2007). De acuerdo a este planteo, la constancia debería ser estudiada vuelo a vuelo (desde una flor de "partida" hacia otra flor de "llegada") determinando cual es la elección que le sigue a una especie vegetal en cualquier contexto, para poder detallar este aspecto del comportamiento (Waser, 1986; Raine \& Chittka, 2007). Además de estas explicaciones vinculadas a limitaciones cognitivas, se ha planteado la constancia floral como una estrategia de forrajeo eficiente en sí misma si la especie floral provee una recompensa de calidad y la obtención de información sobre alternativas es potencialmente costosa (Chittka et al., 1999; Grüter \& Ratnieks, 2011). Por otro lado, la elección del recurso a explotar depende de la historia de cada pecoreadora (el recurso más frecuente en el parche que está pecoreando) (Waser, 1986; Goulson, 2000; Raine \& Chittka, 2005) y las características morfológicas de los individuos (tamaño, largo de la probóscide, entre otros) (Rotheray et al., 2017) que también incidirán en la elección de los recursos a utilizar pues a algunos abejorros les resulta más fácil la obtención de néctar y/o polen (Heinrich 2004; De Luca et al., 2019). En estos ápidos existe una gran variación individual a nivel intra e interespecífico, lo que representa una gran ventaja ya que disminuye la competencia permitiendo que cada pecoreadora pueda explotar un recurso exitosamente sin interferir en el éxito de las demás (Heinrich, 2004; Raine \& Chittka, 2005; Rotheray et al., 2017).

El objetivo de este estudio fue estudiar la constancia floral de los abejorros nativos $B$. pauloensis y $B$. bellicosus en condiciones de campo. 


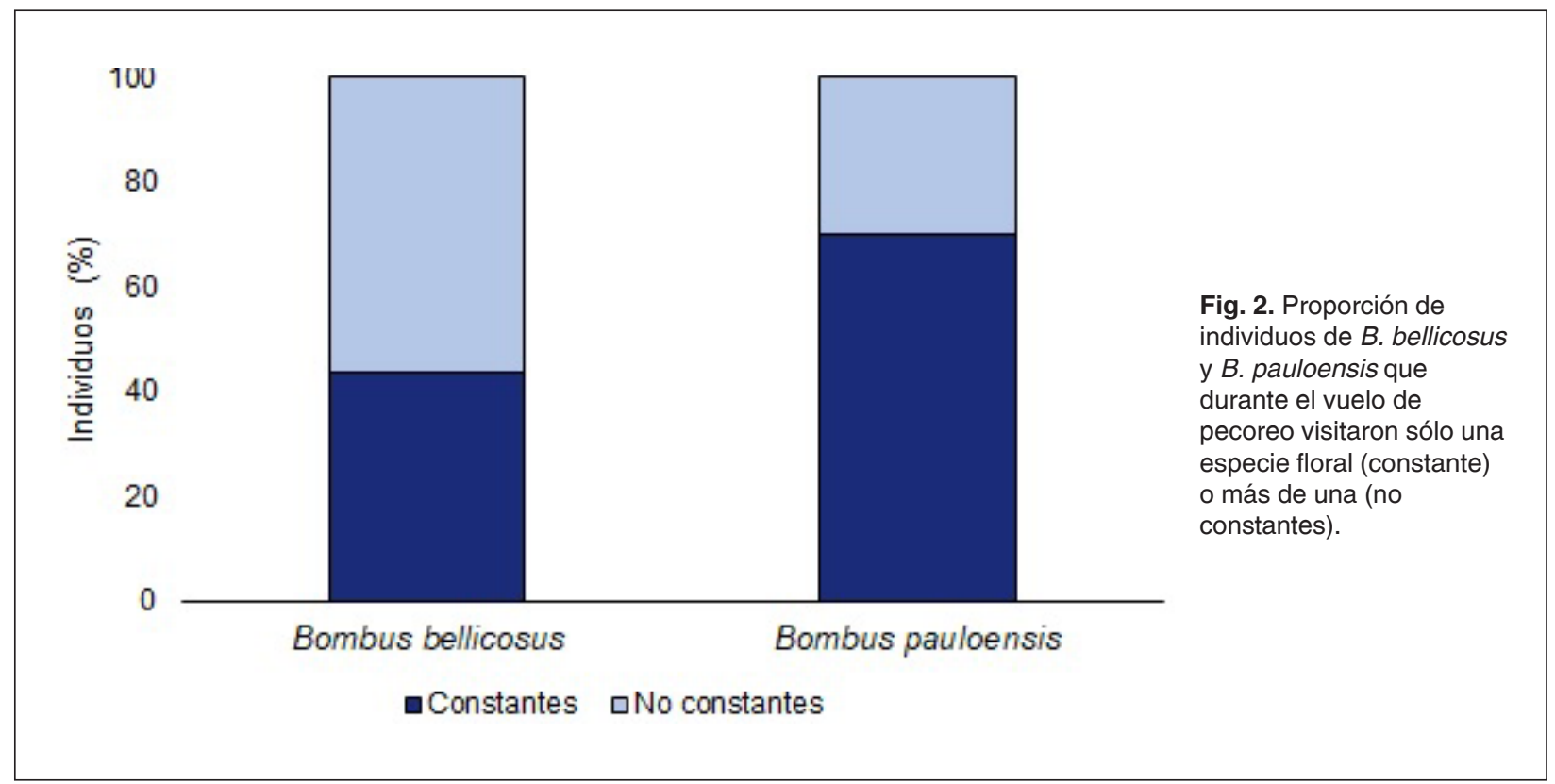

\section{MATERIALES Y MÉTODOS}

El estudio se realizó en la localidad de Semillero en el departamento de Colonia (34029'58.28"S, 57066'99.81"O). El comportamiento de los abejorros fue registrado en condiciones de campo mientras pecoreaban en una pradera combinada compuesta principalmente por trébol rojo (Trifolium pratense), lotus (Lotus corniculatus) y en menor proporción, trébol blanco (Trifolium repens) (Fig. 1). Estas especies forrajeras, solas o en combinación, son comúnmente utilizadas en Uruguay para alimentación del ganado y es muy frecuente observar abejorros recolectando néctar y/o polen de ellas. Durante las observaciones, se identificaron individuos de $B$. pauloensis y $B$. bellicosus, y se registró la secuencia de especies florales que visitaban durante su viaje de pecoreo. Se puso énfasis en que el seguimiento durara el mayor tiempo posible antes de perder de vista a los insectos. Los datos obtenidos fueron analizados de dos maneras distintas. Por un lado, se analizó la constancia de los individuos que visitaron como mínimo 20 unidades florales individuales (UFI, inflorescencias para el trébol rojo y trébol blanco, flor para lotus). En el caso que haya realizado por lo menos un cambio durante el período de observación ese individuo se consideró no constante. Por otro lado, siguiendo el criterio de Raine \& Chittka (2007), se tomó como unidad de análisis el vuelo independiente, de UFI a UFI. Aquí se consideró un vuelo constante cuando el individuo partía de una UFI y volaba hacia otra (flor de llegada) de la misma especie; si el destino era una UFI de una especie diferente ese vuelo se consideró no constante.

\section{Análisis de datos}

Los datos fueron analizados empleando la prueba de Chi-cuadrado, excepto en aquellos casos donde los valores eran muy pequeños donde se recurrió a la prueba de Fisher. Se utilizó el programa estadístico SAS (Statistical Analysis System) (SAS, 2006).

\section{RESULTADOS}

Se analizó la constancia floral de 23 individuos de $B$. bellicosus y 37 de $B$. pauloensis, que visitaron como mínimo 20 unidades florales individuales (UFI). Del total de ejemplares de $B$. bellicosus el $43.5 \%$ visitaron consecutivamente una única especie floral, mientras que para $B$. pauloensis la proporción de individuos constantes fue de $70 \%$ (Fig. 2). Dichos resultados revelan diferencias interespecíficas en el comportamiento de ambas especies, siendo $B$. pauloensis significativamente más constante que $B$. bellicosus $\left(\chi^{2}=55.83 ; \mathrm{P}<0.001\right)$.

Para el estudio de los vuelos de UFI a UFI se analizaron 26 seguimientos de $B$. bellicosus y 38 de B. pauloensis de los cuales se obtuvieron 1505 y 2128 vuelos, respectivamente (Fig. 3). La proporción de vuelos constantes fue significativamente mayor en $B$. pauloensis que en $B$. bellicosus $\left(\chi^{2}=4.24\right.$; $\left.P<0.05\right)$, aunque ambas especies presentaron un alto porcentaje 


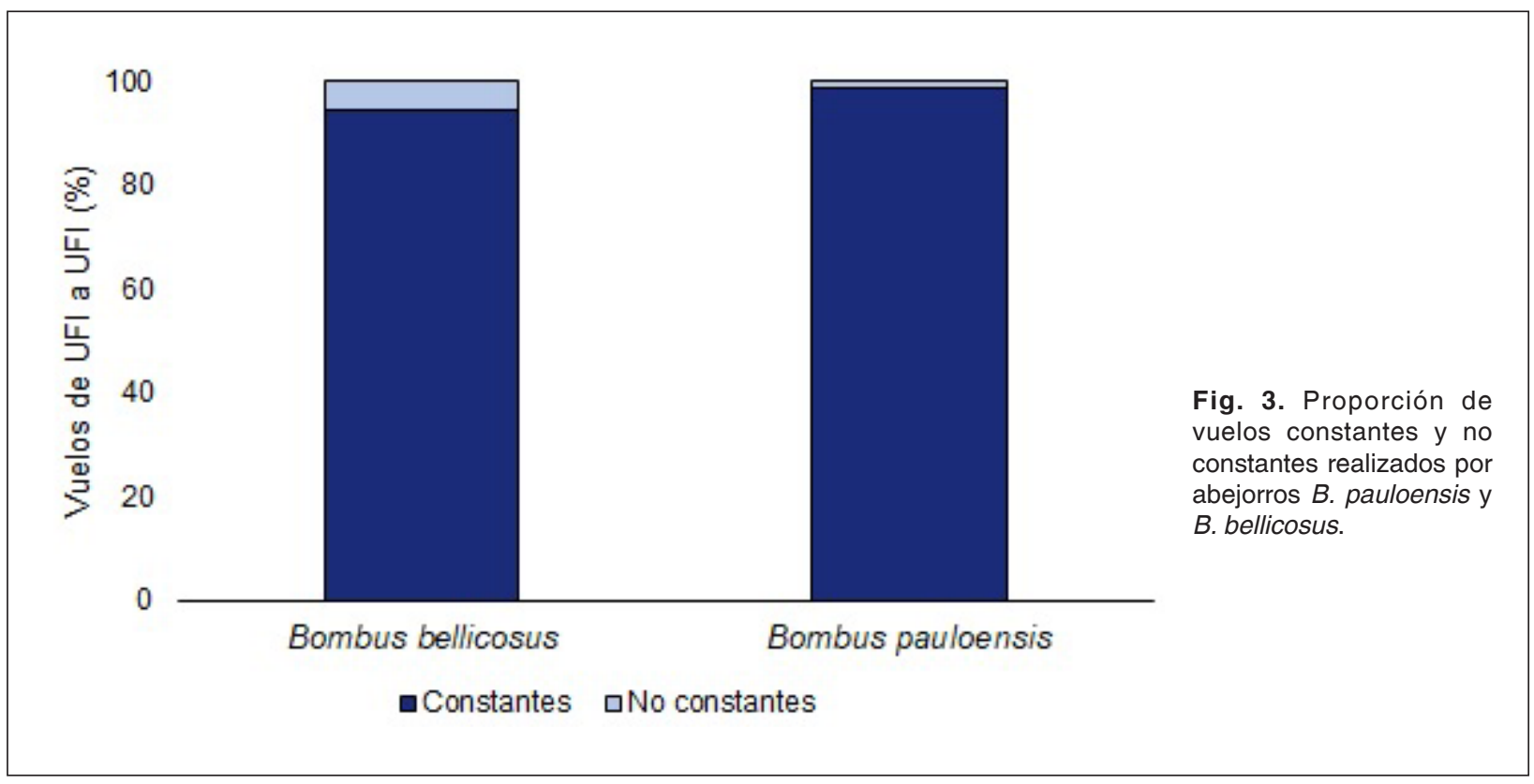

de los mismos (98.7\% y $94.4 \%$, respectivamente).

Los vuelos de UFI a UFI fueron analizados teniendo en cuenta la especie vegetal de partida y la de llegada (Fig. 4). La proporción de vuelos constantes fue mayor que la de no constantes tanto para $B$. bellicosus $\left(\chi^{2}=\right.$ $1820 ; \mathrm{P}<0.001)$ como para B. pauloensis $\left(\chi^{2}=2904 ; \mathrm{P}\right.$ $<0.001$ ) para las tres especies vegetales de partida consideradas (trébol rojo, lotus y trébol blanco). En ambas especies de abejorros cuando la especie vegetal de partida fue el lotus o el trébol rojo la proporción de vuelos constantes superó el $90 \%$, aunque son apreciables algunas diferencias entre ellas. En $B$. bellicosus, los vuelos no constantes fueron menores cuando la especie utilizada fue el lotus (4.1\%) que cuando utilizaban el trébol rojo (6.2\%). Contrariamente, en $B$. pauloensis la proporción de vuelos no constantes fue menor cuando partían del trébol rojo $(0.7 \%)$ que cuando lo hacían del lotus (1.5\%). Por otro lado, en ambas especies de abejorros, cuando la especie de partida fue el trébol blanco la proporción de vuelos no constantes superó el 30\%, ocurriendo el cambio tanto hacia el lotus como hacia el trébol rojo (Fig. 4).

\section{DISCUSIÓN}

Los datos obtenidos de registrar las especies florales visitadas por los abejorros $B$. bellicosus y $B$. pauloensis durante un tramo del vuelo de pecoreo se analizaron siguiendo dos criterios. Con ambos análisis $B$. pauloensis presentó mayor constancia floral que $B$. bellicosus. La constancia floral de estas especies ha sido reportada por Rossi et al. (2015) quienes analizaron este comportamiento mediante el análisis palinológico de muestras de néctar y de polen de individuos pecoreando en el campo. En dicho estudio se constató en ambas especies de abejorros que aproximadamente el $80 \%$ de las muestras provenían de una única especie vegetal. En este estudio donde se analizaron seguimientos individuales en los que usualmente sólo se registra una parte del viaje, la especie $B$. bellicosus no alcanzó el $50 \%$ de vuelos constantes. Al analizar los vuelos de UFI a UFI independientemente, el nivel de constancia aumentó y las diferencias interespecíficas disminuyeron notablemente. Esto podría deberse a que en los individuos que visitan más de un recurso floral, los cambios de especie ocurren con muy baja frecuencia. Por otro lado, Chittka et al. (1999) proponen que estas transiciones podrían ser "errores" que cometen los abejorros al reconocer y/o discriminar las especies utilizadas cuando se encuentra entremezclada con otras, pero una explicación alternativa sería que los individuos simplemente cambian de especie floral pero se mantienen constantes visitando consecutivamente el nuevo recurso vegetal cambiando su constancia floral al nuevo recurso (Raine \& Chittka, 2007). Una forma de profundizar en este aspecto sería realizando seguimientos a los abejorros que permitan registrar el viaje de forrajeo completo conjuntamente con análisis palinológico de la carga de ese viaje. Complementariamente, se deberá determinar el grado de constancia floral que los individuos presentan de acuerdo a si estaban recolectando néctar, polen o ambos. En este sentido, se ha reportado en abejorros de $B$. bellicosus y $B$. pauloensis, que se encuentran 

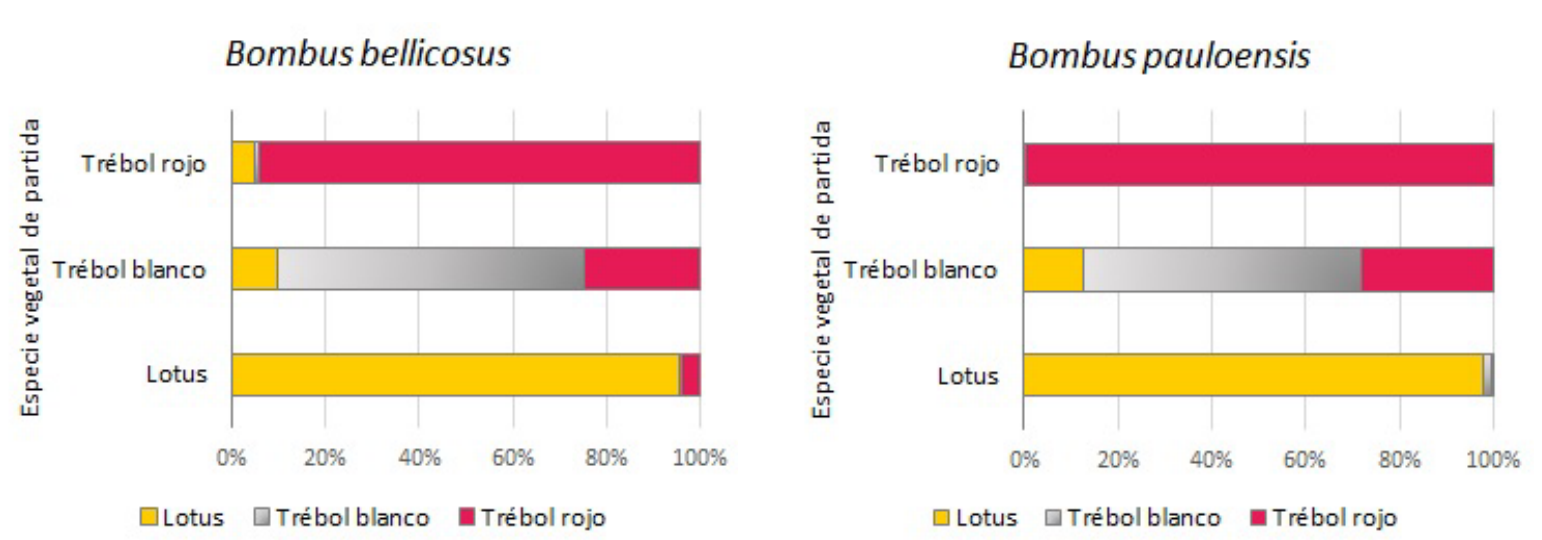

Fig. 4. Proporción de especies florales a las que llegaron los individuos de B. bellicosus y B. pauloensis luego de partir de flores de trébol rojo, trébol blanco y lotus.

recolectando néctar utilizan un mayor número de especies vegetales que las que colectan polen (Rossi et al., 2015; Salvarrey et al., 2020).

La disponibilidad sumado a la recompensa que brindan las distintas especies vegetales son factores determinantes en el grado de constancia floral que exhiben las diferentes especies de abejorros (Waser, 1986). En este estudio, el lotus y el trébol rojo eran los recursos más abundantes y fueron los más explotados por parte de ambas especies de abejorros, siendo el primero más utilizado por $B$. bellicosus y el segundo por $B$. pauloensis. El trébol blanco, que aunque no fue cuantificado se encontraba visiblemente en menor proporción, fue menos visitado. Los individuos que partían del trébol rojo o de lotus posiblemente tenían una alta probabilidad de hallar una flor de la misma especie, lo que reforzaría continuamente la imagen del recurso utilizado y favorecería una mayor constancia floral (Chittka et al., 1997; Goulson, 2000; 2003). Por otro lado, las preferencias observadas por estos recursos coinciden con lo expuesto por Arbulo et al. (2011) quienes en una pradera de similar composición vegetal reportaron la misma repartición de recursos por parte de las dos especies de abejorros. El trébol rojo es una inflorescencia de flores rosadas de corola profunda (7 a $12.5 \mathrm{~mm}$ ) y el néctar es secretado en la base de la corola por lo que su obtención está determinada por el largo de la probóscide del polinizador que la visite (Free, 1970). El lotus por su parte, presenta sus flores amarillas dispuestas en racimos (4-6) y sus corolas son más abiertas y menos profundas $(10 \mathrm{~mm})$ (Izaguirre \& Beyhaut, 1997). Con esto, el trébol rojo sería más fácil de manejar por parte de $B$. pauloensis que posee una probóscide de mayor longitud $(8.4 \mathrm{~mm})$ que $B$. bellicosus $(7.9 \mathrm{~mm})$ que en este contexto encontraría en el lotus un recurso más redituable (Arbulo et al.,
2011). Estas preferencias, por sí solas, no explicarían las diferencias interespecíficas en el grado de constancia exhibido por las especies. Por otro lado,el hecho de que la mayoría de los vuelos constantes ocurren cuando los abejorros $B$. pauloensis parten del trébol rojo y $B$. bellicosus cuando parten del lotus, sugiere que la eficiencia en la obtención de recursos y el nivel de recompensa obtenido podrían ser factores importantes en determinar la constancia en este contexto.

En suma, los resultados obtenidos acerca del grado de constancia de los individuos y las diferencias interespecíficas observadas, aunque descriptivos y preliminares, plantean interrogantes sobre diferentes aspectos de este comportamiento y estarían indicando que los factores que determinan la constancia floral en condiciones de campo pueden ser múltiples. Mientras podría estar implicada una correcta asociación entre la imagen del recurso utilizado y la recompensa que representa, también influiría la eficiencia en manipulación de las flores (que se traduce en tiempo y energía). Como plantean Grueter \& Ratnieks (2011), el grado de constancia floral que presentan los abejorros parecería ser un comportamiento adaptativo a través del cual los individuos, a pesar de la existencia de ciertas limitaciones cognitivas, pueden ajustar el grado de constancia a los niveles de recompensa de las diferentes ofertas florales utilizando información acerca de los recursos disponibles, la competencia con otras especies, la eficiencia en la extracción del néctar y el polen, entre otras.. Se requiere profundizar en estos aspectos a través de estudios de campo así como trabajos experimentales que midan $\mathrm{y} / \mathrm{o}$ controlen distintas variables involucradas (e.g.: abundancias de especies vegetales, recompensas ofrecidas, ausencia de competidores, etc.). 


\section{BIBLIOGRAFÍA}

Arbulo N., Santos E., Salvarrey S. \& C. Invernizzi. 2011. Proboscis Length and Resource Utilization in Two Uruguayan Bumblebees: Bombus atratus Franklin and Bombus bellicosus Smith (Hymenoptera: Apidae). Neotropical Entomology, 40(1): 72-77.

Ashman T.L., Knight T.M., Steet J.R., Burd M. \& D.R. Campbell. 2004. Pollen limitation of plant reproduction: ecological and evolutionary causes and consequences. Ecology 85, 2408-2421.

Chittka L., Gumbert A. \& J. Kunze. 1997. Foraging dynamics of bumble bees: correlates of movement within and between plant species. Behavior Ecology, 8(3):239.

Chittka L., Thomson J. \& N. Waser. 1999. Flower Constancy, Insect Psychology, and Plant Evolution. Naturwissenschaften Review Articles, 86: 361-377.

De Luca P.A., Buchmann S., Galen C., Mason A.C. \& M. Vallejo-Marín. 2019. Does body size predict the buzz-pollination frequencies used by bees? Ecology and Evolution, 9(8):4875-87.

Free J.B. 1970. The flower constancy of bumblebees. Journal of Animal Ecology, 39: 395-402.

Gegear R.J. \& T.M. Laverty. 2005. Flower constancy in bumblebees: a test of the trait variability hypothesis. Animal Behaviour, 69: 939-49.

Goulson D. 2000. Are insects flower constant because they use search images to find flowers? Oikos, 88, 547-552.

Goulson D. 2003. Bumblebees: their behaviour and ecology. Oxford University Press, UK.

Grüter C. \& F.L. Ratnieks. 2011. Flower constancy in insect pollinators: Adaptive foraging behaviour or cognitive limitation? Communicative Integrate Biology, 4:633.

Heinrich B. 2004. Bumblebee Economics. Harvard University Press, Cambridge, Massachusetts, USA.

Hill P.S.M., Hollis J. \& H. Wells. 2001. Foraging decisions in nectarivores: unexpected interactions between flower constancy and energetic rewards. Animal Behaviour, 2001; 62:729-37.

Izaguirre P \& R. Beyhaut. 1997. Las leguminosas en Uruguay y regiones vecinas: Parte 1 Papilionoideae (Faboideae). Montevideo: Hemisferio Sur.

Klein A.M., Vaissière B.E., Cane J.H., Steffan D.I., Cunningham S.A., Kremen C. \& T. Tscharntke. 2007. Importance of pollinators in changing landscapes for world crops. Proceeding of the Royal Society, 274: 303-313.

Laverty T.M. 1994. Costs to foraging bumblebees of switching plant species. Canadian Journal of Zoology, 72: 43-47.

Michener C.D. 2007. The bees of the world. The Johns Hopkins University Press (2nd ed.). Baltimore, Maryland, USA.
Raine N.E. \& L. Chittka. 2005. Comparison of flower constancy and foraging performance in three bumblebee species (Hymenoptera: Apidae: Bombus). Entomology General, 28(2):081-089.

Raine N.E \& L. Chittka. 2007. Flower constancy and memory dynamic in bumblebees (Hymenoptera: Apoidea: Bombus). Entomologia Generalis, 29 (2-4):179-199.

Rossi N., Santos E., Salvarrey S., Arbulo N. \& C. Invernizzi. 2015. Determination of Flower Constancy in Bombus atratus Franklin and Bombus bellicosus Smith (Hymenoptera: Apidae) through Palynological Analysis of Nectar and Corbicular Pollen Loads. Neotropical Entomology, 44(6):546-52.

Rotheray E.L., Osborne J.L. \& D. Goulson D. 2017. Quantifying the food requirements and effects of food stress on bumble bee colony development. Journal of Apicultural Research, 56(3):288-299.

Santos E., Arbulo N., Salvarrey S. \& C. Invernizzi. 2017. Distribución de las especies del género Bombus Latreille (Hymenoptera, Apidae) en Uruguay. Revista de la Sociedad Entomológica Argentina, 76(1-2):22-7.

Salvarrey S., Arbulo N., Rossi C., Santos E., Salvarrey L. \& C. Invernizzi. 2017. Utilización de abejorros nativos (Bombus atratus Franklin y Bombus bellicosus Smith) para mejorar la producción de semillas del trébol rojo (Trifolium pratense). Agrociencia Uruguay, 21:95-104.

Salvarrey S. 2020. Utilización de abejorros nativos como polinizadores de trébol rojo (Trifolium pratense) y tomate (Solanum lycopersicum). Tesis doctoral presentada para la obtención del título Doctor en Ciencias Agrarias en Facultad de Agronomía, Universidad de la República, Montevideo, Uruguay.

Salvarrey S., Santos E., Arbulo N., Giménez G. \& C. Invernizzi. 2020. Characteristics of the tomato fruit (Solanum lycopersicum) using native bumblebees (Bombus atratus) as pollinators in green- house. Agrociencia Uruguay, 24(1):101.

SAS institute Inc. 2006. SAS/Procedures guide versión SAS 9.1.3. SAS Institute Inc., Cary, NC. USA.

Waser N.M. 1986. Flower constancy: definition, cause and measurement. The American Naturalist, 127(5): 593-603.

Waser N.M. \& L.A. Real. 1979. Effective mutualism between sequentially flowering plant species. Nature 281, 670-672.

Wiegmann D.D, Wiegmann D.A \& F.A. Waldron. 2003. Effect of a reward downshift on the consummatory behavior and flower choices of bumblebee foragers. Physiology \& Behavior, 79: 561-566.

Fecha de Recepción: 31 de agosto de 2020 Fecha de Aceptación: 17 de diciembre 2020 